\title{
Assessment of 3D Codes for Predicting Liner Attenuation in Flow Ducts
}

\author{
W. R. Watson, D. M. Nark, and M. G. Jones ${ }^{\ddagger}$ \\ NASA Langley Research Center, Hampton, Virginia 23681-2199, USA
}

\begin{abstract}
This paper presents comparisons of seven propagation codes for predicting liner attenuation in ducts with flow. The selected codes span the spectrum of methods available (finite element, parabolic approximation, and pseudo-time domain) and are collectively representative of the state-of-art in the liner industry. These codes are included because they have two-dimensional and three-dimensional versions and can be exported to NASA's Columbia Supercomputer. The basic assumptions, governing differential equations, boundary conditions, and numerical methods underlying each code are briefly reviewed and an assessment is performed based on two predefined metrics. The two metrics used in the assessment are the accuracy of the predicted attenuation and the amount of wall clock time to predict the attenuation. The assessment is performed over a range of frequencies, mean flow rates, and grazing flow liner impedances commonly used in the liner industry. The primary conclusions of the study are (1) predicted attenuations are in good agreement for rigid wall ducts, (2) the majority of codes compare well to each other and to approximate results from mode theory for soft wall ducts, (3) most codes compare well to measured data on a statistical basis, (4) only the finite element codes with cubic Hermite polynomials capture extremely large attenuations, and (5) wall clock time increases by an order of magnitude or more are observed for a three-dimensional code relative to the corresponding two-dimensional version of the same code.
\end{abstract}

\section{Introduction}

$\mathrm{H}$ GH levels of engine fan noise radiated from modern turbofan engines threaten to curtail much needed growth in commercial air transportation systems worldwide. Engine fan noise is acoustic energy generated at the fan or exit guide vanes of a turbofan engine that radiates forward via the nacelle inlet duct and rearward by propagating through the aft nacelle duct and then refracting through the ambient shear layer. Figure 1 shows a schematic of a typical aircraft

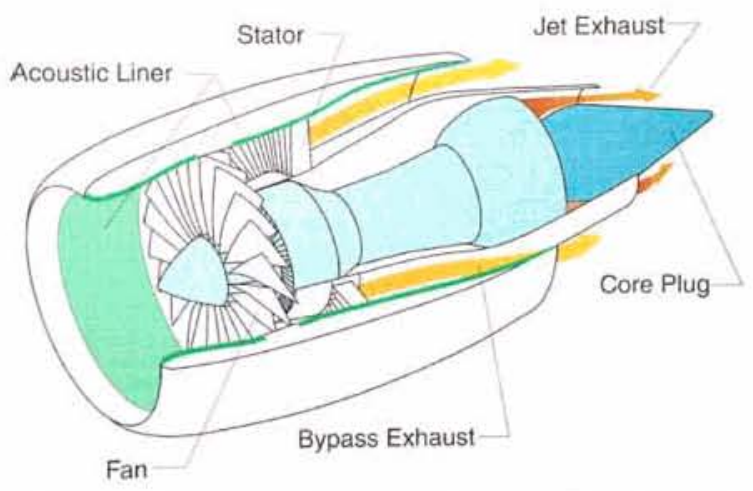

Fig. 1 Schematic of a turbofan engine nacelle with wall lining.

engine nacelle. Earlier versions of the turbofan engine were dominated by tones associated with the blade passage frequency and its harmonics. However, in recent engines the fan blade shapes were modified to incorporate lean and

*Senior Research Scientist, Research and Technology Directorate, Computational AeroSciences Branch, Liner Physics Group, Mail Stop 128, Willie.R.Watson@NASA.Gov. AIAA Associate Fellow.

${ }^{\dagger}$ Research Scientist, Research and Technology Directorate, Structural Acoustics Branch, Liner Physics Group. Mail Stop 463, Douglas.M.Nark@NASA.Gov. Member AIAA.

${ }^{\ddagger}$ Senior Research Scientist, Research and Technology Directorate, Structural Acoustics Branch, Liner Physics Group, Mail Stop 463, Michael.G.Jones@NASA.Gov.AIAA Associate Fellow. 
sweep, such that the blade passage frequency is less dominant. Newer engines have also incorporated increased fan bypass to increase the thrust to drag ratio and fuel efficiency.

One of the most effective means for reducing levels of noise radiating from commercial aircraft engines has been the installation of acoustic treatment in the walls of the inlet and aft fan ducts. Such treatments reduce radiated noise by attenuating inlet and aft fan noise as it propagates through the engine nacelle. Suitable aircraft noise prediction codes are required to optimally design the acoustic treatment for maximum attenuation within a specified nacelle geometry. Time domain methods are a topic of considerable research interest; however, the frequency domain method remains the primary basis for the prediction of liner attenuation in the aircraft noise industry. Although engine designs incorporate three-dimensional (3D) geometries, a literature survey reveals that until the turn of the 21st century, the only numerical method for predicting liner attenuations in 3D geometries was the parabolic approximation. ${ }^{1}$ Since that time, the finite element method $^{2}$ and the pseudo-time domain method $^{3}$ have also emerged. In addition, the NASA Langley Research Center has two finite element $\operatorname{codes}^{4-6}$ that were recently extended to 3D.

Each computer code that has been written based on the above three methods has advantages and disadvantages depending upon the problem being modeled, assumptions that are made, and computer facilities available to code users. Scientists desiring to use codes based on the above three methods are often frustrated because (1) information available to code users is of ten fragmented and incomplete, and much of the theory underlying code development (e.g., the assumptions made, the governing differential equations, the boundary conditions, and the numerical method) is often not clear, (2) comparison of wall clock time statistics between the various codes are not available because codes have not been run for the same problem, using the same computer, and on identical grids, (3) exact analytical and approximate attenuations from mode theory are available to compare with code predictions, but these comparisons are rare, (4) comparison of code predictions with measured data is at best questionable because experiments are seldom designed to provide the necessary code inputs (e.g., the sound source, grazing flow liner impedance, and duct termination conditions), and (5) the measured data contain uncertainties in the inputs needed for code predictions; however, code users have treated these inputs as deterministic.

The purposes of this paper are to (1) collect the most commonly used 3D duct propagation codes (non-vortical and vortical) for predicting liner attenuation under a single umbrella, (2) briefly review the basic assumptions, governing differential equations, boundary conditions, and numerical method underlying code development, and (3) perform an assessment based upon two predefined metrics. The selected codes span the spectrum of methods currently in use (parabolic approximation, finite element method, and pseudo-time domain method) and are collectively representative of the current state-of-art in the liner industry. The two metrics used in this assessment are the accuracy of the predicted attenuation and the wall clock time required to predict the attenuation.

The following section (i.e., section II) describes the physical duct acoustic problem of interest and the basis for code selection. Sections III and IV present the basic assumptions, the governing differential equations, duct boundary conditions, and numerical method underlying code development for both the non-vortical and vortical duct propagation codes. The method used to predict liner attenuation in the presence of grazing flow is described in section V. Results of the code assessment are presented in section VI, and a brief summary along with the primary conclusions of this study are presented in the final section of this paper, section VII.

\section{Statement of Problem and Basis for Code Selection}

$\mathrm{F}$ IGURE 1 shows a sketch of a typical 3D duct geometry. The duct carries a non-uniform, subsonic mean flow from left to right. A Cartesian coordinate system $(z, x, y)$ is used, in which the axial coordinate is $z$ and the two transverse coordinates are $x$ and $y$. The source plane is denoted by an imaginary plane, $\Gamma_{s}$. Two types of acoustic propagation are considered. For inlet propagation, the sound propagates upstream of the fan against the flow and exits the duct as shown in the upper portion of Fig. 2. In this case, the duct termination is located at the entrance to the inlet and is denoted by the plane, $\Gamma_{t}$. The second type of propagation is aft-duct propagation, in which the sound propagates downstream of the fan face and exits the duct via the aft-fan nacelle duct as shown in the lower portion of Fig. 2. For aft propagation, the termination plane, $\Gamma_{t}$, is located downstream of the fan face in the exhaust duct. In both inlet and aft duct propagation, the computational volume will be denoted by $\Omega$ and the perimeter of the duct containing the physical duct walls will be denoted by $\Gamma_{w}$. Let the surface bounding $\Omega$ be denoted by $\Gamma$, such that the union of $\Gamma_{s}, \Gamma_{t}$, and $\Gamma_{w}$ is $\Gamma$

$$
\Gamma=\Gamma_{s} \cup \Gamma_{t} \cup \Gamma_{w}
$$

The perimeter of the duct is lined with acoustic material that is assumed to be locally reacting (i.e., acoustic waves propagate through it normal to the surface), and the normalized acoustic impedance of the wall lining (generally a function of wall location) will be denoted by $\zeta$. Throughout this study, all impedances are normalized with respect 


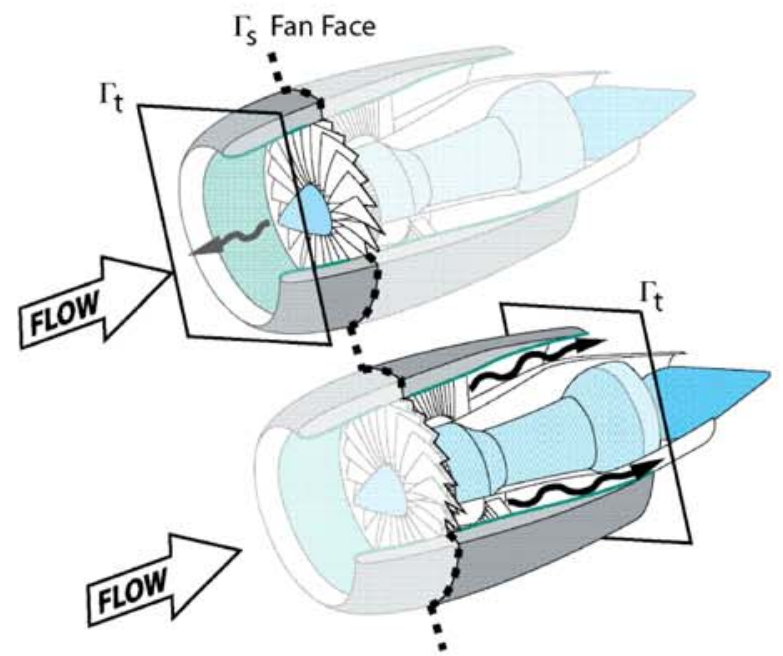

Fig. 2 Schematic of inlet and aft-duct propagation and termination boundary, $\Gamma_{t}$.

to the characteristic impedance of the air in the duct. The problem at hand is to predict the sound attenuation in the presence of the wall lining and grazing flow, given the mean flow field, sound source, normalized wall impedance, and the duct termination condition.

Codes available for predicting the attenuation in a 3D duct, such as is illustrated in Fig. 1, have generally been divided into non-vortical and vortical duct propagation codes. In this study, a suite of seven codes (four non-vortical and three vortical) is selected. The primary basis for code selection was that a two-dimensional (2D) and 3D version of the code be available. This decision was made so that the increase in wall clock time in transitioning from a $2 \mathrm{D}$ version to a 3D version of the same code could be accurately recorded. Although the Actran $\operatorname{code}^{2}$ has a 2D and $3 \mathrm{D}$ version, it is a commercial code and our current license agreement forbids our version to be exported to NASA's Columbia Supercomputer. For this reason, Actran has not been included in the suite of selected codes.

\section{Non-vortical Duct Propagation Codes}

$\mathrm{T}$

HE following section gives a brief overview of the basic assumptions, governing differential equations, boundary conditions, and numerical methods underlying development of the non-vortical duct propagation codes.

\section{A. Assumptions, Governing Equation, and Boundary Conditions}

The basic assumptions underlying the non-vortical duct propagation codes are (1) the acoustic field in the duct is linear, (2) the fluid in the duct is inviscid and irrotational, (3) the fluid is an ideal gas, and (4) the acoustic pressure and density are homentropically related. These assumptions lead to a scalar wave equation (i.e., a convected Helmholtz equation) on the acoustic velocity potential, ${ }^{1,2,7}$

$$
-\frac{D}{D t}\left(\frac{\rho_{0}}{c_{0}^{2}} \frac{D \phi}{D t}\right)+\vec{\nabla} \bullet\left(\rho_{0} \vec{\nabla} \phi\right)-\frac{\rho_{0}}{c_{0}^{2}}\left(\vec{\nabla} \bullet \vec{u}_{0}\right) \frac{D \phi}{D t}=0
$$

where a time dependence of the form $e^{i \omega t}$ has been assumed, $c_{0}$ is the sound speed, $\rho_{0}$ is the mean static density, $\bullet$ denotes a dot product, $\vec{u}_{0}$ is the irrotational mean velocity vector, $\vec{\nabla}$ is the gradient operator, $i$ is the unit imaginary number, and the material derivative is expressed in the frequency domain as

$$
\frac{D}{D t}=i \omega+\vec{u}_{0} \bullet \vec{\nabla}
$$

Upon obtaining the acoustic velocity potential, $\phi$, it is post-processed to obtain the acoustic pressure, $p$, and acoustic particle velocity field, $\vec{u}$

$$
p=-\rho_{0} \frac{D \phi}{D t}, \quad \vec{u}=\vec{\nabla} \phi
$$


The scalar wave equation [Eq. (2)] requires three types of boundary conditions. One boundary condition is required at the source boundary, a second is required at the duct termination, and a third is needed along all rigid or acoustically lined wall segments.

The source plane boundary condition is

$$
p=p_{s}, \quad \text { on } \Gamma_{\mathrm{s}}
$$

where $p_{s}$ is the known source plane acoustic pressure. At the duct termination, the boundary condition is specified in the form

$$
\{p\}=\left[\zeta_{t}\right]\left\{\rho_{0} c_{0} \vec{u} \bullet \vec{n}\right\}, \text { along } \Gamma_{\mathbf{t}}
$$

where $\left[\zeta_{t}\right]$ is the dimensionless node impedance matrix ${ }^{8}$ at points along $\Gamma_{t}, \vec{n}$ is the outward unit normal vector to $\Gamma_{t}$, and $\{p\},\{\vec{\nabla} u \bullet \vec{n}\}$ are vectors containing the acoustic pressure and the normal component of acoustic particle velocity at nodes along $\Gamma_{t}$. Elements in the node impedance matrix, [ $\left.\zeta_{t}\right]$, may be chosen so that the termination is reflecting or nonreflecting. Further, the choice for elements in $\left[\zeta_{t}\right]$ will be discussed in more detail in the results section of this report.

There has been considerable discussion in the literature during the past half century concerning the acoustic boundary condition at an acoustically treated duct wall with a general shape and mean flow. However, the Myers boundary condition $^{9}$ has been generally accepted as correct in modern duct acoustic literature. Therefore, we choose to only include codes that implement the wall impedance boundary condition developed by Myers. ${ }^{9}$ When written in terms of the primitive variables (i.e., the acoustic pressure and the acoustic particle velocity vector), this boundary condition is

$$
-\rho_{0} c_{0} \vec{u} \bullet \vec{n}=-\frac{p}{\zeta}-\frac{\rho_{0} c_{0}}{i \omega}\left(\vec{u}_{0} \bullet \vec{\nabla}\right)\left(\frac{p}{\rho_{0} c_{0} \zeta}\right)+\frac{p}{i \omega \zeta} \vec{n} \bullet \vec{n} \bullet \vec{\nabla} \vec{u}_{0}=0 \text { on } \Gamma_{w}
$$

Equation (7) is restricted to locally-reacting acoustic liners, and will not be valid for those liners that allow wave propagation through the material in directions parallel to the flow path. It is also valid along a rigid wall segment of duct, provided the normalized wall impedance is set to infinity (i.e., $\zeta=\infty$ ). Note that the sound source [Eq. (5)], duct termination [Eq. (6)], and wall impedance [Eq. (7)] boundary conditions have been written in terms of the primitive variables so that they are consistent with the vortical flow boundary conditions that are presented in a later section. However, these boundary conditions [Eqs. (5)-(7)] are easily transformed to boundary conditions on the acoustic velocity potential by substitution from Eq. (4).

\section{B. Numerical Methods}

It remains a formidable task to obtain solutions to the convected Helmholtz equation [Eq. (2)], coupled with the sound source, duct termination, and wall impedance boundary conditions. Exact solutions to this equation for arbitrary boundary condition parameters, and means flows have thus far not been found. However, the invention of large-scale digital computers has allowed numerical solutions to be obtained. These numerical solutions are essential for optimum design of noise efficient aircraft. Over the past two decades, two methods for solving the 3D scalar wave equation (the convected Helmholtz equation), coupled to the sound source, duct termination, and wall impedance boundary conditions, have emerged. These two methods are the parabolic approximation and the finite element method. Dougherty ${ }^{1}$ originally proposed the parabolic approximation for duct aeroacoustics and Watson ${ }^{11}$ initially proposed the finite element methodology presented in this study. Although the original application of the finite element methodology ${ }^{11}$ was for 2D impedance eduction in uniform flows, the method has recently been extended to 3D rectangular geometries and non-uniform mean flows. ${ }^{5}$ The purpose of the following subsections is to discuss these two numerical methods (i.e., the parabolic approximation and the finite element method) along with their salient features and limitations. Readers are reminded that the details presented in this and subsequent sections are provided as a quick review and more technical detail is available in the original publications. ${ }^{1,5,6}$

\section{The Parabolic Approximation}

Recently, a fan noise duct propagation and radiation $\operatorname{code}^{12}$ was developed that utilizes a parabolic approximation to the scalar wave equation defined by Eq. (2). The code is composed of five distinct modules: (1) input/output specification, (2) fluid dynamics/acoustic grid generation, (3) background flow calculation, (4) acoustic propagation, and (5) acoustic radiation. The acoustic propagation module is the focus of this section, and it utilizes a parabolic approximation to the scalar wave equation. ${ }^{1,13}$ This approach affords very efficient propagation calculations, allowing for complex 3D geometries to be handled with relatively low computational costs. This efficiency comes at the 
expense of reduced accuracy as the direction of propagation of an acoustic mode diverges from the preferred angle of the parabolic approximation. Additionally, loss of accuracy may occur when reflection of acoustic waves in the axial direction becomes important, as these effects are not captured in this formulation. Nevertheless, if appropriate care is taken to account for these limitations, the parabolic approximation can provide an efficient and useful environment to perform 3D computations.

Although the parabolic approximation and general splitting technique were discussed in detail in previous work, ${ }^{5}$ a brief review is provided for the sake of completeness. The full formulation is based on a solution to Eq. (2) in 3D geometries. However, for simplicity, a rectangular duct is considered in which a uniform axial mean flow is present. In this situation, Eq. (2) is rewritten in the form

$$
-\left(1-M_{0}^{2}\right) \frac{\partial \phi}{\partial z^{2}}=\nabla_{\perp}^{2} \phi-2 i k M_{0} \frac{\partial \phi}{\partial z}+k^{2} \phi
$$

where $\nabla_{\perp}, M_{0}$, and $k$ are the transverse Laplacian, mean flow Mach number, and freespace wave number, respectively

$$
\nabla_{\perp}^{2}=\nabla_{\perp} \bullet \nabla_{\perp}=\frac{\partial^{2}}{\partial x^{2}}+\frac{\partial^{2}}{\partial y^{2}}, \quad M_{0}=\frac{u_{0}}{c_{0}}, \quad k=\frac{\omega}{c_{0}}
$$

Here, $u_{0}$ is the uniform axial flow velocity and an $e^{-i \omega t}$ time dependence is assumed. As discussed by Dougherty ${ }^{13}$ and outlined by Corones, ${ }^{14}$ a derivation based on splitting the solution of Eq. (2) into 'positive' traveling (i.e., $\phi^{+}$) and 'negative' traveling (i.e., $\phi^{-}$) waves is proposed. This general splitting is represented as

$$
\left\{\begin{array}{c}
\phi^{+} \\
\phi^{-}
\end{array}\right\}=[T]\left\{\begin{array}{c}
\phi \\
\frac{\partial \phi}{\partial z}
\end{array}\right\} \text { or }\left\{\begin{array}{c}
\phi \\
\frac{\partial \phi}{\partial z}
\end{array}\right\}=[T]^{-1}\left\{\begin{array}{c}
\phi^{+} \\
\phi^{-}
\end{array}\right\}
$$

where the splitting matrix, $[T]$, is arbitrary. Substitution of the general variable splitting given by Eq. (10) into Eq. (8) leads to a coupled pair of differential equations for $\phi^{ \pm}$of the form

$$
\begin{gathered}
\left(1-M_{0}^{2}\right) \frac{\partial}{\partial z}\left\{\begin{array}{l}
\phi^{+} \\
\phi^{-}
\end{array}\right\}= \\
{\left[\left(1-M_{0}^{2}\right)\left(\frac{\partial T}{\partial z}\right)[T]^{-1}+[T]\left[\begin{array}{cc}
0 & 1-M_{0}^{2} \\
-\left(\nabla_{\perp}^{2}+k^{2}\right) & 2 i k M_{0}+M_{0} \frac{\partial M_{0}}{\partial z}
\end{array}\right][T]^{-1}\right]\left\{\begin{array}{l}
\phi^{+} \\
\phi^{-}
\end{array}\right\}}
\end{gathered}
$$

Dougherty ${ }^{1}$ considered plane waves to produce a splitting of the form

$$
\left\{\begin{array}{c}
\phi \\
\frac{\partial \phi}{\partial z}
\end{array}\right\}=\left[\begin{array}{cc}
1 & 1 \\
\frac{-i k}{1+M_{0}} & \frac{i k}{1-M_{0}}
\end{array}\right]\left\{\begin{array}{l}
\phi^{+} \\
\phi^{-}
\end{array}\right\}
$$

Typically, at this point in the derivation, the terms that couple the equations for $\phi^{ \pm}$are neglected to produce a single parabolic equation for the 'positive' or 'negative' traveling wave.

The general formulation outlined by Dougherty considers a system of orthogonal, curvilinear coordinates $\left(\xi_{1}, \xi_{2}, \xi_{3}\right)$, defined so that $\xi_{3}$ is aligned with the steady potential mean flow. Following the aforementioned splitting process and neglecting the off-diagonal terms in the resulting matrix equation gives the parabolic approximation

$$
\frac{\partial \phi^{+}}{\partial \xi_{3}}=\frac{h_{3} i k}{1+M_{0}} \phi^{+}-\frac{h_{3}}{2 i k} \nabla_{\perp}^{2} \phi^{+}-\frac{1}{2 h_{1} h_{2}} \frac{\partial}{\partial \xi_{3}}\left(h_{1} h_{2}\right) \phi^{+}
$$

where $h_{i}$ is the element of length given by

$$
h_{i}=\sqrt{\left(\sum_{j=1}^{3} \frac{\partial x_{j}}{\partial \xi_{i}}\right)^{2}} \text { for } i=1,2,3 .
$$

The source boundary condition is given by

$$
\phi^{+}=\phi_{s}, \quad \text { on } \Gamma_{\mathrm{s}}
$$

where, $\phi_{s}$ is the known source potential. The solution for $\phi^{+}$is marched from the source to the duct termination and no termination boundary condition is required, as the $\phi^{ \pm}$coupling is neglected. 
The wall impedance boundary condition is also only approximately satisfied. This is achieved by assuming that the acoustic pressure and the normal component of acoustic particle velocity are continuous across an infinitely thin boundary layer. Denoting quantities within the duct interior with subscript 1 and those below the infinitely thin boundary layer with subscript 2 , the impedance boundary condition below the boundary layer may be written as

$$
\pm \frac{1}{h_{j}} \frac{\partial \phi_{2}^{+}}{\partial \xi_{j}}=\frac{i k \phi_{2}^{+}}{\zeta}
$$

where $\phi_{2}^{+}$is the acoustic velocity potential below the boundary layer and the sign is taken as negative for minimum $\xi_{j}$ and positive for maximum $\xi_{j}$. The continuity of pressure condition leads to

$$
\frac{D \phi_{1}^{+}}{\bar{D} t}=-i \omega \phi_{2}^{+}, \quad \frac{D}{\bar{D} t}=\left[-i \omega+\vec{u}_{0} \bullet \vec{\nabla}\right]
$$

where $\vec{u}_{0}$ is the mean flow velocity and the normal particle displacement condition gives

$$
\left(\frac{D}{\bar{D} t}\right)^{-1} \vec{\nabla} \phi_{1}^{+} \cdot \vec{n}=\frac{i}{\omega} \vec{\nabla} \phi_{2}^{+} \bullet \vec{n}
$$

Finally, eliminating $\phi_{2}^{+}$and neglecting derivatives of the Mach number in the direction of the flow leads to

$$
\frac{\partial \phi^{+}}{\partial \xi_{j}}= \pm \frac{i k}{\zeta} \frac{h_{j}}{\left(1+M_{0}\right)^{2}} \phi^{+} \mp \frac{h_{j} M_{0}}{1+M_{0}} \frac{\partial}{\partial \xi_{3}}\left(\frac{1}{\zeta}\right) \phi^{+}
$$

The parabolic equation [Eq. (13)] and associated boundary conditions [Eqs. (14) and (15)] can be efficiently solved using a marching method that advances the solution in the $\xi_{3}$ direction. The classical fourth order Runge-Kutta method is well suited to this purpose and is used because of its accuracy and ease of implementation. The transverse derivatives can be evaluated by any number of numerical schemes (e.g., finite difference, finite element). In the current implementation, a pseudospectral approach is used, in which the values of $\phi^{+}$are approximated at the grid points by a series of sine and cosine functions.

Let $\xi_{1}$ and $\xi_{2}$ be discretized on uniformly-spaced grids taken from 0 to $\pi$. To approximate the transverse derivative of $\phi^{+}$with respect to $\xi_{1}$, the wave is modeled at each discrete $\xi_{2}$ value by

$$
\phi^{+}\left(\xi_{1}, \xi_{2}\right)=A_{1}\left(\xi_{2}\right) \sin \left(\xi_{1}\right)+A_{2}\left(\xi_{2}\right) \sin \left(2 \xi_{1}\right)+\sum_{I_{1}=0}^{N_{1}-1} B_{I_{1}}\left(\xi_{2}\right) \cos \left(I_{1} \xi_{1}\right)
$$

where $A_{1}, A_{2}$, and $B_{I_{1}}$ are modal coefficients and $N_{1}$ is the maximum grid index number in the $\xi_{1}$ direction. The wall impedance boundary condition from Eq. (15) is used to determine the coefficients $A_{1}$ and $A_{2}$, and a fast Fourier transform method is used to evaluate the $B_{I_{1}}$ coefficients. Once these coefficients are determined, the derivatives are calculated from

$$
\frac{\partial \phi^{+}}{\partial \xi_{1}}=A_{1}\left(\xi_{2}\right) \cos \left(\xi_{1}\right)+2 A_{2}\left(\xi_{2}\right) \cos \left(2 \xi_{1}\right)-\sum_{I_{1}=0}^{N_{1}-1} I_{1} B_{I_{1}}\left(\xi_{2}\right) \sin \left(I_{1} \xi_{1}\right)
$$

and

$$
\frac{\partial^{2} \phi^{+}}{\partial \xi_{1}^{2}}=-A_{1}\left(\xi_{2}\right) \sin \left(\xi_{1}\right)-4 A_{2}\left(\xi_{2}\right) \sin \left(2 \xi_{1}\right)-\sum_{I_{1}=0}^{N_{1}-1} I_{1}^{2} B_{I_{1}}\left(\xi_{2}\right) \cos \left(I_{1} \xi_{1}\right)
$$

The same process is followed to evaluate the derivatives in the $\xi_{2}$ direction. Substituting the transverse derivatives into Eq. (13) completes the evaluation of the right-hand side and the solution is marched forward to obtain $\phi^{+}$.

\section{The Finite Element Method}

This section describes the weak, finite element solution to the convected Helmholtz equation [Eq. (2)] that satisfies the source, duct termination, and wall impedance boundary condition. To begin, the weak, Galerkin, finite element approximate solution to the acoustic potential, $\phi$, requires that the acoustic potential be first approximated by a class of complete basis functions, $N_{I}$,

$$
\phi=\sum_{I=1}^{N N} N_{I} \Phi_{I}
$$


where $\Phi_{I}$ is a set of generalized coordinates whose relationship to $\phi$ will be determined and the basis functions are understood to be function of the spatial coordinates, $(z, x, y)$. The residual error, $R_{1}$, resulting from the substitution of Eq. (19) into the governing differential equation [Eq. (2)] is now minimized, by requiring that it be orthogonal to each of the basis functions, $N_{J}$

$$
\int_{\Omega} R_{1} N_{J} d \Omega=0
$$

and the residual error is

$$
R_{1}=-\frac{D}{D t}\left(\frac{\rho_{0}}{c_{0}^{2}} \frac{D \phi}{D t}\right)+\vec{\nabla} \bullet\left(\rho_{0} \vec{\nabla} \phi\right)-\frac{\rho_{0}}{c_{0}^{2}}\left(\vec{\nabla} \bullet \vec{u}_{0}\right) \frac{D \phi}{D t}
$$

Equation (20) is a system of $N N$ linear equations in $N N$ unknown generalized coordinates, $\Phi_{J}$. The weak formulation is achieved by applying Green's theorem to the second derivative terms in Eq. (20), and reducing them to first order. For example,

$$
\int_{\Omega} \vec{\nabla} \bullet\left(\rho_{0} \vec{\nabla} \phi\right) N_{J} d \Omega=-\int_{\Omega} \rho_{0}(\vec{\nabla} \phi \bullet \vec{n})\left(\vec{\nabla} N_{J} \bullet \vec{n}\right) d \Omega+\int_{\Gamma} \rho_{0} \vec{\nabla} \phi \bullet \vec{n} N_{J} d \Gamma
$$

Recalling that, $\vec{u} \bullet \vec{n}=\vec{\nabla} \phi \bullet \vec{n}$ [see Eq. (4)], gives

$$
\int_{\Omega} \vec{\nabla} \bullet\left(\rho_{0} \vec{\nabla} \phi\right) N_{J} d \Omega=-\int_{\Omega} \rho_{0}(\vec{\nabla} \phi \bullet \vec{n})\left(\vec{\nabla} N_{J} \bullet \vec{n}\right) d \Omega+\int_{\Gamma} \rho_{0} \vec{u} \bullet \vec{n} N_{J} d \Gamma
$$

Substituting the termination [Eq. (6)] and wall impedance [Eq. (7)] boundary conditions into the surface integral in Eq. (23), followed by substitution of Eq. (23) into Eq. (20), allows Eq. (20) to be written in weak form.

The system of $N N$ linear equations in $N N$ unknowns resulting from the weak form of Eq. (20) is constructed using a 3D finite element methodology. The finite element methodology used in this paper guarantees continuity of the acoustic potential and the acoustic particle velocity vector (i.e., $\vec{\nabla} \phi$ ) within and on the boundaries of $\Omega$. The 3D finite element method assumes that there are $N$ grid lines in the axial or $z$ direction, $M$ grid lines in the $x$ direction, and $Q$ grid lines in the $y$ direction of the duct. As shown in Fig 3., grid lines need not be equally spaced and an element is identified by a triple index notation, $[I, J, K]$. A typical element, $[I, J, K]$, is bounded on the right and left by the grid lines, $y=y_{K}$, and, $y=y_{K+1}$, on the bottom and top by grid lines, $x=x_{J}$, and, $x=x_{J+1}$, and on the front and back by grid lines, $z=z_{I}$, and, $z=z_{I+1}$. Brick elements with eight nodes are used in the analysis. A typical brick element is

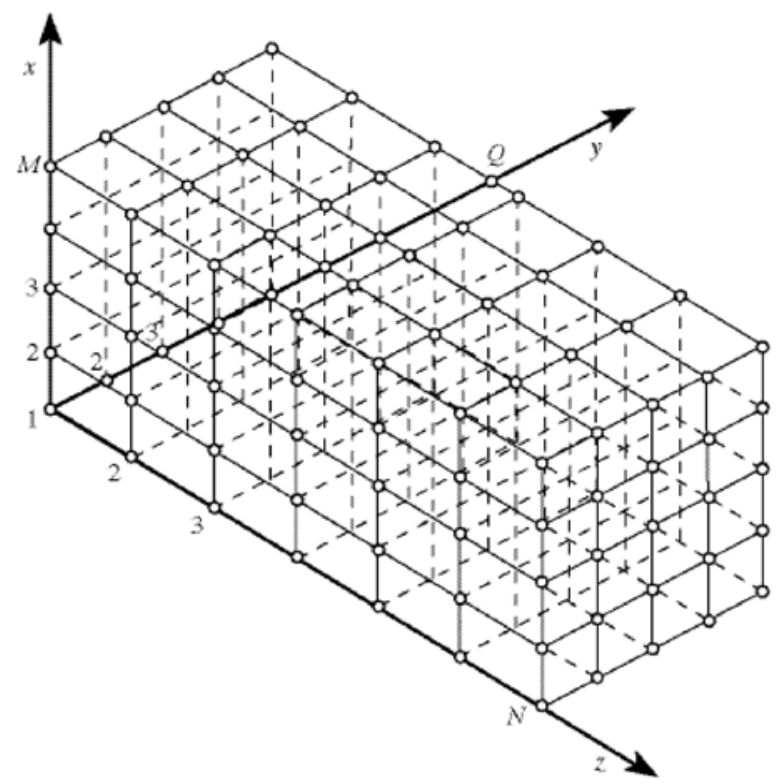

Fig. 3 Finite-element discretization of 3D duct.

shown in Fig 4. Each brick element, $[I, J, K]$, consists of eight local node numbers labeled $1,2, \ldots 8$. Each element 


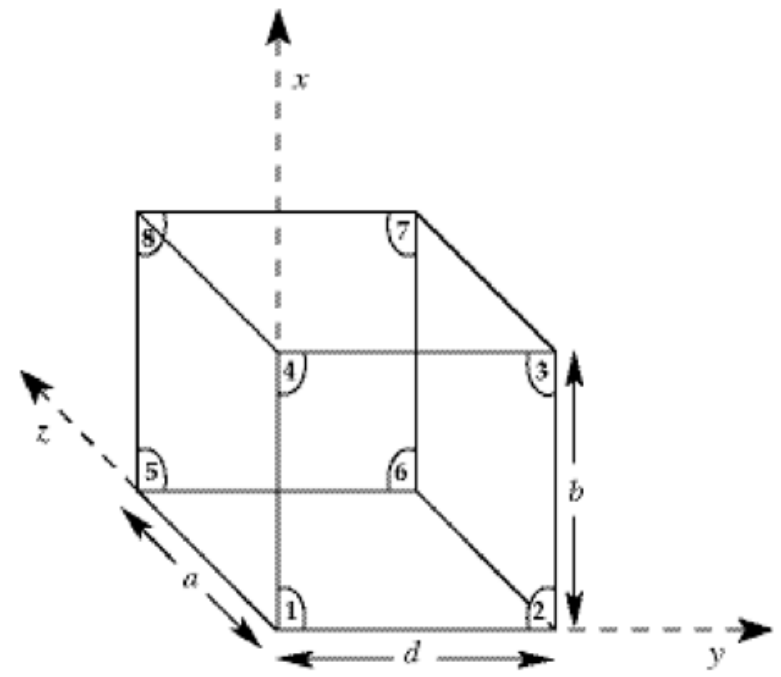

Fig. 4 A Typical 3D finite-element brick element and local node numbering system.

is considered to have length $a$, height $b$, and width $d$, as shown. The objective of the 3D finite element method is to obtain the unknown acoustic velocity potential and its derivatives at the nodes of each of the $(N-1)(M-1)(Q-1)$ elements. Within each element, $\phi$ is represented as linear combinations of sixty-four, 3D, cubic, Hermite polynomials, $N_{1}, N_{2}, N_{3} \ldots N_{64}$ that comprise a complete set of basis functions within each element

$$
\phi=\sum_{I=1}^{I=64} N_{I} \Phi_{I}
$$

The finite element analysis allows the sixty-four 3D basis functions, $N_{I}$, to be easily constructed from the products of the four 1D cubic Hermite polynomial basis functions

$$
\begin{array}{ll}
f_{1}(z, a)=1-3(z / a)^{2}+2(z / a)^{3}, & f_{2}(z, a)=z(z / a-1)^{2} \\
f_{3}(z, a)=(z / a)^{2}(3-2 z / a), & f_{4}(z, a)=\left(z^{2} / a\right)(z / a-1)
\end{array}
$$

in the three coordinate directions. For example, it is easily shown that

$$
N_{1}=f_{1}(z, a) f_{1}(x, b) f_{1}(y, d), N_{2}=f_{2}(z, a) f_{1}(x, b) f_{1}(y, d), \ldots N_{64}=f_{4}(z, a) f_{4}(x, b) f_{4}(y, d)
$$

Similarly, the relationship between the unknown generalized coordinates, $\Phi_{I}$, and the velocity potential at each node points, $\left(z_{I}, x_{J}, y_{K}\right)$, is easily obtained. For example,

$$
\Phi_{1}=\phi\left(z_{I}, x_{J}, y_{K}\right), \Phi_{2}=\frac{\partial \phi\left(z_{I}, x_{J}, y_{K}\right)}{\partial z}, \ldots \Phi_{64}=\frac{\partial^{3} \phi\left(z_{I+1}, x_{J+1}, y_{K+1}\right)}{\partial z \partial x \partial y}
$$

Substituting Eqs. (24) into Eq. (20), using Green's theorem to reduce the second order derivatives to first order derivatives, and then substituting the wall impedance and duct termination boundary conditions into the surface integrals, allows one to compute the contributions to the minimization of the total residual error from each individual element

$$
\int_{\Omega} R_{1} N_{J} d \Omega=\sum_{==1}^{N-1} \sum_{J=1}^{M-1} \sum_{K=1}^{Q-1}\left[A^{I, J, K}\right]\left\{\Phi^{[I, J, K]}\right\}
$$

where the sum on the right-hand side of Eq. (28) is performed using the usual rules of finite element assembly. In Eq. (28), $\left[A^{[I, J, K]}\right]$ is a $64 \times 64$ complex matrix for each element, $[I, J, K]$, and $\left\{\Phi^{[I, J, K]}\right\}$ is a $64 \times 1$ column vector containing the unknown acoustic potential and its derivatives at the eight nodes of the element. The coefficients in the local stiffness matrix, $\left[A^{[I, J, K]}\right]$, are computed in closed form using the Mathematica intrinsic functions. ${ }^{15}$ 
Assembly of the global equations for the computational domain is a basic procedure in the finite-element method. Appropriate shifting of rows and columns is all that is required to add the local element matrix, $\left[A^{[I, J, K]}\right]$, directly into the global matrix, $\left[A_{f}\right] .{ }^{16}$ This process leads to a set of linear matrix equations of the form

$$
\left[A_{f}\right]\{\Phi\}=\{0\}
$$

where $\left[A_{f}\right]$ is a complex, block tridiagonal matrix whose order is $8 M N Q$, and $\{\Phi\}$ and $\{0\}$ are $8 M N Q \times 1$ column vectors. The vector $\{\Phi\}$ contains the nodal values of the unknown acoustic potential and its derivatives at the nodes of the elements and $\{0\}$ is the null vector. The matrix $\left[A_{f}\right]$ is singular until the source conditions are applied.

The source is introduced into Eq. (29) by constraining the nodal degrees of freedom at the source plane in the usual manner. ${ }^{16}$ After implementation of the source condition, Eq. (29) remains block tridiagonal and is of the form

$$
[A]\{\Phi\}=\{F\}
$$

where $\{F\}$ now contains the source effects and $[A]$ is a modified form of $\left[A_{f}\right]$ with the same structure. Much practical importance arises from the matrix structure in Eq. (30), as it is convenient for minimizing storage and maximizing computational efficiency. Special matrix techniques exist for a solution of this structure. An unsymmetrical parallel sparse solver with equation reordering to minimize fill is used to decompose $[A]$ into the products of a unit lower triangular, a unit upper triangular matrix, and a nonsingular diagonal matrix. The sequential operations of forward and backward substitution are used to obtain the solution vector, $\{\Phi\}$. All computations are performed on a parallel computer and only the nonzero elements in $[A]$ are stored and operated upon during the solution stage.

\section{Vortical Duct Propagation Codes}

$\mathrm{T}$ HE following section gives a brief overview of the basic assumptions, governing differential equations, boundary conditions, and numerical methods underlying development of the vortical duct propagation codes.

\section{A. Assumptions, Governing Equations, and Boundary Conditions}

The vortical flow duct propagation codes are based on the same assumption as that for non-vortical flow, except that the irrotationality assumption is not imposed. Consequently, there is not an acoustic velocity potential and the solution is obtained in terms of the primitive variables that satisfy the sound source, wall impedance, and duct termination boundary conditions. The basic assumptions underlying code development are that (1) the acoustic field is linear, (2) the fluid flowing in the duct is inviscid, (3) the fluid is an ideal gas, and (4) the acoustic pressure and density remain homentropically related.

Using the above assumptions, the linearized equation that governs conservation of mass in a flow duct with an inviscid fluid is ${ }^{3,4}$

$$
c_{0}^{2} \frac{D}{D t}\left(\frac{p}{c_{0}^{2}}\right)+\gamma p_{0} \vec{\nabla} \bullet \vec{u}+p \vec{\nabla} \bullet \vec{u}_{0}+c_{0}^{2} \gamma(\vec{u} \bullet \vec{\nabla}) \frac{p_{0}}{c_{0}^{2}}=0
$$

and the linearized equations that govern the conservation of momentum is

$$
\gamma p_{0} \frac{D \vec{u}}{D t}+\gamma p_{0}(\vec{u} \bullet \vec{\nabla}) \vec{u}_{0}+p \frac{D \vec{u}_{0}}{D t}+c_{0}^{2} \vec{\nabla} p=0
$$

where $p_{0}$ is the mean static pressure, and $\gamma$ is the ratio of the specific heat at constant pressure to that at constant volume. The acoustic particle velocity vector, $\vec{u}$, is assumed to contain components $u, v$, and $w$ in the $x, y$, and $z$ directions, respectively. In 3D, the linearized continuity equation [Eqs. (31)] is a scalar equation and Eq. (32) is a vector equation with three components. Thus, the linearized mass and momentum equations are a system of four equations in four unknowns (i.e., $p, u, v$, and $w$ ) that can be solved uniquely to determine the unknown variables that satisfy the sound source, duct termination, and wall impedance boundary conditions.

In the current subsonic flow problem, the linearized acoustic system in three space dimensions requires three boundary conditions at the source boundary, one boundary condition at the duct termination, and another boundary condition at either a rigid or acoustically lined wall segment. ${ }^{17}$ The wall impedance boundary condition remains the Myers boundary condition [Eq. (7)], and we will specify the acoustic pressure and the transverse components of the acoustic particle velocity at the source boundary as

$$
\left\{\begin{array}{l}
p \\
u \\
v
\end{array}\right\}=\left\{\begin{array}{l}
p_{s} \\
u_{s} \\
v_{s}
\end{array}\right\} \text { on } \Gamma_{\mathrm{s}}
$$


where $p_{s}, u_{s}$ and $v_{s}$ are the known values of $p, u$ and $v$ at points along $\Gamma_{s}$. Finally, at the duct termination, the boundary condition remains that given by Eq. (6).

\section{B. Numerical Methods}

Four vortical flow, duct propagation codes, are included in the current suite. Each code solves Eqs. (31) and (32), and satisfies the sound source [Eqs. (33)], duct termination [Eq. (6)], and wall impedance [Eqs. (7)] boundary conditions. The first two codes are based upon a finite element solution methodology, and were initially developed at the NASA Langley Research Center in 2006. ${ }^{4}$ The second two codes use a pseudo-time marching approach, and were developed at the Boeing aircraft company with partial funding from NASA in $2006 .^{3}$ The following subsections give a brief description of the numerical methods underlying the development of the vortical flow, duct propagation codes.

\section{The Finite Element Method}

The two vortical, finite element, duct propagation codes solve Eqs. (31) and (32) using a finite element methodology. The numerical procedure parallels that used in section 2 . However, only linear basis functions are used and the wall and exit impedance boundary conditions are satisfied by constraining the nodal degrees of freedom. To begin, the Galerkin, finite element approximate solution to the linearized mass and momentum equations, requires that each primitive variable be first approximated by a class of complete basis functions, $N_{I}$,

$$
p=\sum_{I=1}^{N N} N_{I} p_{I}, u=\sum_{I=1}^{N N} N_{I} u_{I}, v=\sum_{I=1}^{N N} N_{I} v_{I}, w=\sum_{i=1}^{N N} N_{I} w_{I}
$$

where $p_{I}, u_{I}, v_{I}$ and $w_{I}$ are generalized coordinates. The residual errors, $R_{c}$ and $\left\{R_{m}\right\}$ resulting from the substitution of Eq. (34) into the linearized mass and momentum equations [Eqs. (31) and (32)] are now minimized, by requiring that they be orthogonal to each of the basis functions, $N_{J}$

$$
\begin{gathered}
\int_{\Omega} R_{c} N_{J} d \Omega=0 \\
\int_{\Omega}\left\{R_{m}\right\} N_{J} d \Omega=0 \\
R_{c}=c_{0}^{2} \frac{D}{D t}\left(\frac{p}{c_{0}^{2}}\right)+\gamma p_{0} \vec{\nabla} \bullet \vec{u}+p \vec{\nabla} \bullet \vec{u}_{0}+c_{0}^{2} \gamma(\vec{u} \bullet \vec{\nabla}) \frac{p_{0}}{c_{0}^{2}} \\
\left\{R_{m}\right\}=\left\{\gamma p_{0} \frac{D \vec{u}}{D t}+\gamma p_{0}(\vec{u} \bullet \vec{\nabla}) \vec{u}_{0}+p \frac{D \vec{u}_{0}}{D t}+c_{0}^{2} \vec{\nabla} p\right\}
\end{gathered}
$$

The residual error, $R_{c}$, is a scalar, while the residual error, $\left\{R_{m}\right\}$, is a vector (with three components)

$$
\left\{R_{m}\right\}=\left\{\begin{array}{l}
R_{m z} \\
R_{m x} \\
R_{m y}
\end{array}\right\}
$$

where $R_{m z}, R_{m x}$ and $R_{m y}$ are the residual error components in the $z, x$ and $y$ directions, respectively, of the linearized momentum equation. Equations (35) and (36) reduce to a system of $4 N N$ linear equations in $4 N N$ unknown generalized coordinates, $p_{I}, u_{I}, v_{I}$ and $w_{I}$.

The system of $4 N N$ linear equations and $4 N N$ unknown generalized coordinates is constructed using a 3D finite element method similar to that used for the convected Helmholtz equation. The 3D finite element method assumes that there are $N$ nodes in the axial or $z$ direction, $M$ nodes in the $x$ direction, and $Q$ nodes in the $y$ direction of the duct, just as in Fig. 3. Brick elements with eight nodes are again used (Fig. 4) and the objective of the 3D finite element method is to obtain the solution for the four unknown primitive variables at the nodes of each of the $(N-1)(M-1)(Q-1)$ elements. Within each element, each primitive variable is represented as a linear combinations of eight, 3D, linear polynomials, $N_{1}, N_{2}, N_{3} \ldots N_{8}$ that comprise a complete set of basis functions within each element. Just as with the 
scalar wave equation, the finite element method allows the eight 3D basis functions, $N_{I}$, and generalized coordinates $p_{I}, u_{I}, v_{I}$, and $w_{I}$ to be easily constructed from products of the two linear polynomial basis functions

$$
f_{1}(z, a)=1-z / a, \quad f_{2}(z, a)=z / a
$$

in each of the three coordinate directions, along with the requirement that the primitive variables each be continuous within $\Omega$ and along inter-element boundaries. For example, it can be shown that

$$
N_{1}=f_{1}(z, a) f_{1}(x, b) f_{1}(y, d), N_{2}=f_{1}(z, a) f_{1}(x, b) f_{2}(y, d), \ldots N_{8}=f_{2}(z, a) f_{1}(x, b) f_{1}(y, d)
$$

and that each generalized coordinate, $p_{I}, u_{I}, v_{I}$ and $w_{I}$ are therefore the values of $p, u, v$ and $w$ at local node $I$. Substituting the series expansions in Eq. (34) into Eqs. (35) and (36) allows one to compute the contributions to the minimization of the residual from each individual element

$$
\int_{\Omega}\left\{\begin{array}{l}
R_{C} \\
R_{m z} \\
R_{m x} \\
R_{m y}
\end{array}\right\} N_{J} d \Omega=\sum_{I=1}^{N-1} \sum_{J=1}^{M-1} \sum_{K=1}^{Q-1}\left[A^{I, J, K}\right]\left\{\Phi^{[I, J, K]}\right\}
$$

where the sum on the right-hand side of Eq. (42) is again performed using the usual rules of finite element assembly. In Eq. (42), $\left[A^{[I, J, K]}\right]$ is a $32 \times 32$ complex matrix for each element, $[I, J, K]$, and $\left\{\Phi^{[I, J, K]}\right\}$ is a $32 \times 1$ column vector containing the unknown primitive variables at the eight nodes of the element. The coefficients in the local stiffness matrix, $\left[A^{[I, J, K]}\right]$, are again computed in closed form using the Mathematica intrinsic functions. ${ }^{15}$ Assembling the elements for the entire domain results in a matrix equation of the form:

$$
\left[A_{f}\right]\{\Phi\}=\{0\}
$$

where $\left[A_{f}\right]$ is a complex, block tridiagonal matrix, whose order is $4 M N Q$, and $\{\Phi\}$ and $\{0\}$ are $4 M N Q \times 1$ column vectors. The vector $\{\Phi\}$ contains the nodal values of the primitive variables at the nodes of the elements and $\{0\}$ is the null vector.

Before a non-trivial solution to Eq. (43) can be obtained, source, termination plane, and wall impedance boundary conditions must be imposed. To satisfy the source, wall impedance, and termination plane boundary conditions, the residual error corresponding to these boundary conditions is minimized [i.e., after substitution from Eq. (34)]. For example, to satisfy the source boundary condition, the source residual, $R_{s}$, is minimized

$$
\begin{aligned}
& \int_{\Gamma_{s}} R_{S} N_{J} d \Gamma=0 \\
& R_{s}=\left\{\begin{array}{l}
p-p_{s} \\
u-u_{s} \\
v-v_{s}
\end{array}\right\}
\end{aligned}
$$

Equations (44) constitute a set of constraints that are imposed on the solution to Eq. (43). Similarly, to satisfy the wall impedance boundary condition, we minimize the wall residual, $R_{w}$

$$
\begin{gathered}
\int_{\Gamma_{w}} R_{w} N_{J} d \Gamma=0 \\
R_{w}=\rho_{0} c_{0} \vec{u} \bullet \vec{n}-\frac{p}{\zeta}-\frac{\rho_{0} c_{0}}{i \omega}\left(\vec{u}_{0} \bullet \vec{\nabla}\right)\left(\frac{p}{\rho_{0} c_{0} \zeta}\right)+\frac{p}{i \omega \zeta} \vec{n} \bullet \vec{n} \bullet \vec{\nabla}\left(\vec{u}_{0}\right)=0, \text { on } \Gamma_{w}
\end{gathered}
$$

Equations (46) constitute a set of constraints that, when imposed on the solution to Eq. (43), satisfy the wall impedance boundary condition. A similar procedure is used to satisfy the termination boundary condition. The above methodology of introducing the boundary conditions as constraints results in a modified set of matrix equations of the form

$$
[A]\{\Phi\}=\{F\}
$$

where $\{F\}$ contains source effects and $[A]$ has a structure similar to $\left[A_{f}\right]$. Equation (48) is also solved using a parallel band solver to obtain the solution vector, $\{\Phi\}$. 


\section{The Pseudo-Time Method}

A pseudo-time method has also been developed for solutions of Eqs. (31) and (32) when coupled with the source [Eq. (33)], the duct termination [Eq. (6)], and wall impedance [Eq. (7)] boundary conditions. The starting point for the pseudo-time marching code or method is to rewrite Eq. (31) and Eq. (32) in a compact vector format at a location $(z, x, y)$

$$
-i \omega\{Q\}+[A]\left\{\frac{\partial Q}{\partial x}\right\}+[B]\left\{\frac{\partial Q}{\partial y}\right\}+[C]\left\{\frac{\partial Q}{\partial z}\right\}+[D]\{Q\}=0, \quad\{Q\}=\left\{\begin{array}{c}
p \\
u \\
v \\
w
\end{array}\right\}
$$

where $[A],[B]$ and $[C]$ are $4 \times 4$ matrices that contain mean flow effects and $[D]$ is a $4 \times 4$ matrix containing terms related to the gradient of the mean flow field. The coefficients in $[A],[B],[C]$, and $[D]$ are rather lengthy and are not written explicitly in this report. Detailed expressions for these matrices can be found in the original reference. ${ }^{3}$

Adding a pseudo-time derivative to the compact vector form of the linearized equations that govern continuity of mass and momentum [Eq. (49)] gives

$$
\left\{\frac{\partial Q}{\partial \tau}\right\}=-i \omega\{Q\}+[A]\left\{\frac{\partial Q}{\partial x}\right\}+[B]\left\{\frac{\partial Q}{\partial y}\right\}+[C]\left\{\frac{\partial Q}{\partial z}\right\}+[D]\{Q\}
$$

where $\tau$ is a pseudo-time variable. The pseudo-time derivative is introduced so that Eq. (50) may be marched in pseudotime until the solution is steady. Thus, the steady solution to Eqs. (50) is the solution to Eq. (49). Equation (50) is discretized in pseudo-time using a forward-Euler scheme

$$
\left\{\frac{Q^{n+1}-Q^{n}}{\Delta \tau}\right\}+i \omega\left\{Q^{n+1}\right\}=[A]\left\{\frac{\partial Q^{n}}{\partial x}\right\}+[B]\left\{\frac{\partial Q^{n}}{\partial y}\right\}+[C]\left\{\frac{\partial Q^{n}}{\partial z}\right\}+[D]\left\{Q^{n}\right\}
$$

where $n$ is the pseudo-time index, $\Delta \tau$ is the pseudo-time step, and the first term on the right side of Eq. (50) is treated implicitly. Equation (51) is now written in a delta form

$$
\{\Delta Q\}\left[\frac{1}{\Delta \tau}+i \omega\right]=-i \omega\left\{Q^{n}\right\}+[A]\left\{\frac{\partial Q^{n}}{\partial x}\right\}+[B]\left\{\frac{\partial Q^{n}}{\partial y}\right\}+[C]\left\{\frac{\partial Q^{n}}{\partial z}\right\}+[D]\left\{Q^{n}\right\}, \Delta Q=Q^{n+1}-Q^{n}
$$

Upon approximating the spatial derivatives and then implementing the boundary conditions, Eq. (52) can be marched in pseudo-time until the solution is steady (i.e., until $\{\Delta Q\}=\{0\}$ ) to achieve the frequency domain solution. The spatial gradients are approximated using a fourth order optimized finite difference technique, referred to as the dispersionrelation-preserving (DRP) scheme. ${ }^{18}$ The DRP scheme is a central difference approximation to the gradient in each coordinate direction that uses a seven-point stencil. The dispersion relation-preserving scheme is especially designed to minimize dissipation and dispersion errors with a resolution of eight points per wavelength. At and near boundaries of the computational domain, the DRP central difference scheme requires points from outside the computational domain. This is avoided by switching to a fourth order, optimized, one-sided difference at and near these boundaries. Further, an eighth-order filtering scheme is applied to prevent spurious waves from contaminating the solution domain as the solution is advanced forward in pseudo-time. The sound source, wall impedance, and duct termination boundary conditions are inserted into the pseudo-time marching method in strong form. ${ }^{3}$ Other intricate details involved in the implementation of the DRP scheme to the linearized equations of mass and momentum continuity, as well as the implementation of other acoustic boundary conditions not considered in this paper are described in detail in another reference. ${ }^{3}$

The pseudo-time marching method is implemented on a curvilinear mesh using uniform computational coordinates. Thus, solutions using arbitrary geometries are possible. In addition, the pseudo-time marching code uses a domain decomposition scheme that utilizes the message passing interface (MPI) method to reduce wall clock time on parallel computers. The domain decomposition is only in the primary flow direction; thus, the other two dimensions are not parallelized. Therefore, the $2 \mathrm{D}$ version of the pseudo-time marching method is not currently parallelized.

\section{Liner Attenuation}

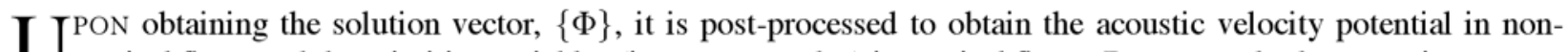
vortical flows and the primitive variables (i.e., $p, u, v$, and $w$ ) in vortical flows. Because only the acoustic pressure 
is readily measurable, it is convenient to use the measured difference in the integrated sound pressure level between the source plane and an axial plane , $z=z_{L}$, to compute the liner attenuation

$$
\begin{gathered}
\triangle S P L=10 \log _{10}\left(\frac{S P L_{s}}{S P L_{z L}}\right) \\
S P L_{s}=\int_{\Gamma_{s}} p p^{*} d \Gamma \\
S P L_{z L}=\int_{z_{L}} p p^{*} d \Gamma
\end{gathered}
$$

where the superscript, $*$, denote the complex conjugate. Equation (53) is used as a measure of liner attenuation throughout this report. The location of the axial plane, $z=z_{L}$ [Eq. (55)], is taken either upstream of the source plane for inlet propagation or downstream of the source plane for aft propagation.

\section{Results and Discussion}

SEVEN duct propagation codes ( 3 non-vortical and 4 vortical) were exported to the Columbia clusters to perform the current assessment. Table 1 gives an overview of the code names (CH2DDS, LEE2DDS, LEE2DIS, CH3DPA,

Table 1. Summary of duct propagation codes exported to the Columbia clusters.

\begin{tabular}{|l|c|l|l|}
\hline \multicolumn{1}{|c|}{ Name } & Dimensionality & Governing Differential Equation & \multicolumn{1}{c|}{ Numerical Method } \\
\hline CH2DDS & 2D & Convected Helmholtz & Cubic Finite Element \\
\hline LEE2DDS & 2D & Linearized Euler & Linear Finite Element \\
\hline LEE2DIS & 2D & Linearized Euler & Pseudo Time-Stepping \\
\hline CH3DPA & 3D & Convected Helmholtz & Parabolic Approximation \\
\hline CH3DDS & 3D & Convected Helmholtz & Cubic Finite Element \\
\hline LEE3DDS & 3D & Linearized Euler & Linear Finite Element \\
\hline LEE3DIS & 3D & Linearized Euler & Pseudo Time-Stepping \\
\hline
\end{tabular}

CH3DDS, LEE3DDS, LEE3DIS), as well as the dimensionality (2D or 3D), governing differential equation (convected Helmholtz or Linearized Euler), and numerical method (parabolic approximation, finite element, or pseudotime domain) underlying the development of each code. Note that the parabolic approximate solution to the convected Helmholtz equation is run only in 3D to limit the number of computer runs and because the 3D and 2D code run at nearly equal speeds. Further, the duct propagation code consisting of a 3D finite element solution to the linearized Euler was not run during this assessment due to time constraints.

Each code is run for a geometry corresponding to the LaRC Grazing Incidence Tube (GIT) ${ }^{11}$ using an assumed uniform mean flow. This geometry and mean flow was chosen because: (1) approximate liner attenuations from mode theory are available for comparisons, (2) experimental data are available to compare with code predictions, and (3) some of the duct propagation codes are currently written for a rectangular geometry such as present in the GIT. The GIT has a square cross-section, with a height and width of $0.051 \mathrm{~m}$. For this study, the distance between source and measurement planes is $0.61 \mathrm{~m}$ (i.e., $z_{L}=0.61 \mathrm{~m}$ ). Sound and flow in the GIT are currently directed in the same direction, so that only aft-duct propagation was modeled in the duct propagation codes. A grid refinement study showed that all codes were nearly converged on a uniform computational grid with 97 points in the axial and 21 points in the two transverse directions for the Mach numbers and frequencies used in this study. Thus, all 3D and 2D codes were run on uniform $97 \times 21 \times 21$ and $97 \times 21$ baseline grids, respectively.

\section{A. Accuracy Assessment}

The most important metric in assessing a propagation code is the accuracy of the predicted attenuation. To assess the accuracy of the predicted attenuation, two example problems were chosen for which the attenuation could be determined analytically. The first example problem tests the ability of the duct propagation codes to predict the attenuation of a plane wave propagating through a hardwall duct with flow. It is well known that a plane wave does not attenuate as it propagates along a hardwall duct, and therefore the codes should predict zero attenuation. The second 
example problem tests the ability of each code to predict the finite attenuation of a cut-on mode in a softwall duct with a uniform lining. For this case, an approximate value for the attenuation is obtained using mode theory.

Table 2. Attenuation in dB of plane wave in a hardwall duct with an anechoic termination

\begin{tabular}{|c|c|c|c|c|c|c|c|}
\hline \multicolumn{8}{|c|}{$M_{0}=0.0$} \\
\hline$f, \mathrm{kHz}$ & Exact & CH2DDS & LEE2DDS & LEE2DIS & CH3DPA & CH3DDS & LEE3DIS \\
\hline 1.0 & 0.00 & 0.00 & 0.01 & 0.00 & 0.00 & 0.00 & 0.00 \\
\hline 2.0 & 0.00 & 0.00 & 0.02 & 0.00 & 0.00 & 0.00 & 0.00 \\
\hline 3.0 & 0.00 & 0.00 & 0.00 & 0.05 & 0.00 & 0.00 & 0.09 \\
\hline \multicolumn{8}{|c|}{$M_{0}=0.3$} \\
\hline 1.0 & 0.00 & 0.00 & 0.01 & 0.00 & 0.00 & 0.00 & 0.00 \\
\hline 2.0 & 0.00 & 0.00 & 0.01 & 0.00 & 0.00 & 0.00 & 0.00 \\
\hline 3.0 & 0.00 & 0.00 & 0.21 & 0.01 & 0.00 & 0.00 & 0.01 \\
\hline \multicolumn{8}{|c|}{$M_{0}=0.5$} \\
\hline 1.0 & 0.00 & 0.00 & 0.00 & 0.00 & 0.00 & 0.00 & 0.00 \\
\hline 2.0 & 0.00 & 0.00 & 0.02 & 0.00 & 0.00 & 0.00 & 0.00 \\
\hline 3.0 & 0.00 & 0.00 & 0.06 & 0.00 & 0.00 & 0.00 & 0.00 \\
\hline
\end{tabular}

In the first example problem, the hardwall attenuations are simulated for nine different conditions obtained from three selected excitation frequencies $(1.0,2.0$, and $3.0 \mathrm{kHz})$ and three selected mean flow Mach numbers $(0.0,0.3$, 0.5 ). For each simulation, the source is planar (i.e., $p_{s}=1$ ), the termination is nonreflecting (i.e. $\left[\zeta_{t}\right]=[I]$ ), and the mean static pressure, density, and temperature are those at standard atmospheric conditions $\left(p_{0}=101325.0 \mathrm{~N} / \mathrm{m}^{2}\right.$, $\left.\rho_{0}=1.2 \mathrm{~kg} / \mathrm{m}^{3}, T_{0}=293.0 \mathrm{~K}\right)$. Here, $[I]$ is the identity matrix. Results are presented in Table 2 . Computer simulations from each of the selected codes are in good agreement with the exact value of zero. There is a small attenuation of 0.21 for LEE2DDS at Mach 0.3 for the highest frequency (i.e., $3.0 \mathrm{kHz}$ ). The discrepancy in this case is apparently due to the use of a low-order approximation (i.e., expansion in terms of linear basis functions) to the acoustic pressure for the highest excitation frequency. When the LEE2DDS grid density was increased by a factor of two, the predicted attenuation was reduced from 0.21 to $0.05 \mathrm{~dB}$.

The second example tests the ability of each code to predict the finite attenuation of a cut-on mode in a softwall duct with a uniform lining [i.e., a duct for which the lower and two sidewalls are hard and the upper wall contains a uniform liner (Impedance values for the softwall are chosen from a database of six production liners that were tested in the GIT. Impedance values were judiciously chosen from the database so that they covered the maximum and minimum ranges of resistance and reactance available in the database of six liners)]. In this case an approximate value for the attenuation can be obtained using mode theory. It is easily shown that the exact value of the sound pressure level attenuation from Eq. (53) for this configuration is

$$
\Delta S P L=20 \log _{10}[e] \Im\left[K_{n} z_{L}\right]
$$

where $\Im\left[\right.$ ] denotes the imaginary part of the complex expression enclosed within the brackets, $z_{L}$ is the location of the downstream plane (i.e., $0.610 \mathrm{~m}$ for the current study), and the axial propagation constant $K_{n}$ satisfies the transcendental equation

$$
i k \alpha_{n}=\zeta \lambda_{n} \tan \left(\lambda_{n} H\right)
$$

where $H$ is the duct height and

$$
\alpha_{n}=1-2\left(\frac{M_{0} K_{n}}{k}\right)+\left(\frac{M_{0} K_{n}}{k}\right)^{2}
$$

The transverse wavenumber, $\lambda_{n}$, and axial propagation constant, $K_{n}$, are related by the dispersion relation

$$
\frac{K_{n}}{k}=\frac{M_{0} \pm \sqrt{1-\left(1-M_{0}^{2}\right)\left(\lambda_{m, n} / k\right)^{2}}}{-\left(1-M_{0}^{2}\right)}
$$

The axial propagation constant, $K_{n}$, is approximated by finding the roots of the transcendental equation [Eq. (57)] using a Newton-Raphson iterative scheme. Note that for a given value of $\lambda_{n}$, the axial propagation constant $K_{n}$ satisfying 
Eq. (59) has two roots. Here, we will choose the root that corresponds to right-moving waves in the duct. It is easily shown that right-moving waves are identified as those waves for which the axial propagation constant, $K_{n}$, has a zero or negative imaginary part and a positive real part, respectively. In addition, the above solution [i.e., Eq. (56)-(59)] considers only the zeroth order spanwise mode so that the solution in the third dimension (i.e., the $y$ dimension) is planar. Thus, the solution for this softwall example problem is only 2D ( $p=p(z, x)$, see Fig. 3), so that both the 2D and 3D version of a code should return identical solutions.

Attenuations due to a softwall were computed for 36 different conditions. These 36 conditions correspond to identical frequencies and Mach numbers as in Table 2. However, four softwall impedances were used with each combination of Mach number and frequency. The sound source, $p_{s}$, was chosen to be the least attenuated mode in the duct, the termination was chosen to be nonreflecting (i.e. $\left[\zeta_{t}\right]=\zeta_{E}[I]$ ), and the mean static pressure, density, and temperature are those at the standard atmospheric conditions used in the hard wall simulations. Here, $\zeta_{E}$ is the characteristic impedance for the least attenuated right-moving wave and was computed from the exact expression for the outgoing wave impedance

$$
\zeta_{E}=\frac{k-M_{0} K_{n}}{K_{n}}
$$

Attenuations predicted from CH2DDS, LEE2DDS, LEE2DIS, CH3DPA and CH3DDS for the softwall duct with an anechoic termination and zero flow are compared to the approximate attenuation from mode theory in Table 3 . The normalized resistance and reactance of the wall lining are given in columns 2 and 3, respectively, of the table. Recall that the sound source in this second example was a softwall duct mode. Results from LEE3DIS were not computed because the available version of LEE3DIS was designed to take source inputs in the form of hardwall duct modes only. As shown in Table 3, CH2DDS, LEE2DDS, CH3DDS, and LEE2DIS are in good agreement with mode theory

Table 3. Attenuation in $\mathrm{dB}$ of lowest mode in a softwall duct with an anechoic termination

\begin{tabular}{|c|c|c|c|c|c|c|c|c|}
\hline $\mathrm{kHz}$ & $\Re[\zeta]$ & $\Im[\zeta]$ & Theory & CH2DDS & LEE2DDS & LEE2DIS & CH3DPA & CH3DDS \\
\hline 1.0 & 0.50 & -0.50 & 61.70 & 61.70 & 59.50 & 58.62 & 68.77 & 61.10 \\
\hline 1.0 & 0.50 & 0.50 & 50.00 & 50.00 & 49.80 & 48.70 & 43.15 & 49.50 \\
\hline 1.0 & 2.00 & -0.50 & 24.60 & 24.60 & 24.40 & 24.89 & 25.73 & 24.30 \\
\hline 1.0 & 2.00 & 0.50 & 24.00 & 24.00 & 23.90 & 23.77 & 24.25 & 23.80 \\
\hline \hline 2.0 & 0.50 & -0.50 & 143.00 & 143.00 & 61.70 & 73.27 & 79.00 & 142.00 \\
\hline 2.0 & 0.50 & 0.50 & 19.30 & 19.30 & 19.20 & 18.13 & 35.52 & 19.10 \\
\hline 2.0 & 2.00 & -0.50 & 26.50 & 26.50 & 26.30 & 26.60 & 25.39 & 26.20 \\
\hline 2.0 & 2.00 & 0.50 & 21.00 & 21.00 & 20.90 & 20.75 & 22.71 & 20.80 \\
\hline \hline 3.0 & 0.50 & -0.50 & 36.10 & 36.10 & 35.90 & 33.88 & 24.06 & 35.70 \\
\hline 3.0 & 0.50 & 0.50 & 10.30 & 10.30 & 10.20 & 9.76 & 8.35 & 10.20 \\
\hline 3.0 & 2.00 & -0.50 & 26.40 & 26.40 & 26.30 & 25.87 & 27.63 & 26.10 \\
\hline 3.0 & 2.00 & 0.50 & 17.90 & 17.90 & 17.70 & 17.35 & 17.63 & 17.70 \\
\hline
\end{tabular}

results with one exception. Note that at $2.0 \mathrm{kHz}$, LEE2DDS and LEE2DIS fails to predict the large attenuation of 143 $\mathrm{dB}$ when the impedance is $\zeta=0.5-0.5 i$. Further, the finite element codes (CH2DDS, CH3DDS, and LEE2DDS) were observed to converge to the mode theory attenuation from below (i.e., the attenuation is slightly lower than that of mode theory on a coarse grid, but gradually increases to the mode theory result as the grid is refined). The failure of three of the codes (LEE2DDS, LEE2DIS and CH3DPA) to predict the large peak attenuation of $143 \mathrm{~dB}$ was somewhat surprising and should be further investigated. LEE2DIS is observed to be slightly less accurate than the duct propagation codes that use a direct solve strategy (CH2DDS, CH3DDS, and LEE2DDS). Given the low fidelity of CH3DPA compared to the other codes in the suite, its performance at frequencies away from the $143 \mathrm{~dB}$ peak is good, except for the $2.0 \mathrm{kHz}$ results when $\zeta=0.5+0.5 i$ and the $3.0 \mathrm{kHz}$ results when $\zeta=0.5-0.5 i$. However, these two frequencies and impedances do correspond to the sources with the widest wave angles where the parabolic approximation is less accurate.

Softwall attenuations predicted at Mach 0.3 and Mach 0.5 are given in Tables 4 and 5, respectively. Several observations are noted for these Mach numbers. A significant reduction in the peak attenuation is observed at $2.0 \mathrm{kHz}$ 
Table 4 Attenuation in dB of lowest mode in a softwall duct with an anechoic termination.

$M_{0}=0.3$

\begin{tabular}{|c|c|c|c|c|c|c|c|c|}
\hline $\mathrm{kHz}$ & $\Re[\zeta]$ & $\Im[\zeta]$ & Theory & CH2DDS & LEE2DDS & LEE2DIS & CH3DPA & CH3DDS \\
\hline 1.0 & 0.50 & -0.50 & 21.20 & 21.20 & 20.80 & 26.90 & 39.76 & 21.00 \\
\hline 1.0 & 0.50 & 0.50 & 44.10 & 44.10 & 44.10 & 37.48 & 29.85 & 43.70 \\
\hline 1.0 & 2.00 & -0.50 & 13.70 & 13.70 & 13.60 & 18.51 & 15.57 & 13.50 \\
\hline 1.0 & 2.00 & 0.50 & 15.00 & 15.00 & 15.00 & 18.79 & 15.03 & 14.90 \\
\hline \hline 2.0 & 0.50 & -0.50 & 39.10 & 39.10 & 40.70 & 66.93 & 43.01 & 38.70 \\
\hline 2.0 & 0.50 & 0.50 & 19.20 & 19.20 & 19.30 & 18.49 & 24.90 & 19.00 \\
\hline 2.0 & 2.00 & -0.50 & 15.20 & 15.20 & 15.10 & 20.41 & 15.31 & 15.00 \\
\hline 2.0 & 2.00 & 0.50 & 14.00 & 14.00 & 14.00 & 17.25 & 14.28 & 13.80 \\
\hline \hline 3.0 & 0.50 & -0.50 & 52.30 & 52.30 & 52.50 & 43.14 & 56.22 & 51.70 \\
\hline 3.0 & 0.50 & 0.50 & 11.50 & 11.50 & 11.70 & 10.51 & 9.78 & 11.40 \\
\hline 3.0 & 2.00 & -0.50 & 15.80 & 15.80 & 15.80 & 20.52 & 16.73 & 15.60 \\
\hline 3.0 & 2.00 & 0.50 & 12.80 & 12.80 & 12.90 & 15.04 & 12.72 & 12.70 \\
\hline
\end{tabular}

Table 5 Attenuation in $\mathrm{dB}$ of lowest mode in a softwall duct with an anechoic termination.

$M_{0}=0.5$

\begin{tabular}{|c|c|c|c|c|c|c|c|c|}
\hline $\mathrm{kHz}$ & $\Re[\zeta]$ & $\Im[\zeta]$ & Theory & CH2DDS & LEE2DDS & LEE2DIS & CH3DPA & CH3DDS \\
\hline 1.0 & 0.50 & -0.50 & 13.30 & 13.30 & 13.00 & 23.57 & 31.12 & 13.10 \\
\hline 1.0 & 0.50 & 0.50 & 38.90 & 39.00 & 39.10 & 41.33 & 25.53 & 38.50 \\
\hline 1.0 & 2.00 & -0.50 & 9.93 & 9.93 & 9.87 & 15.78 & 12.05 & 9.83 \\
\hline 1.0 & 2.00 & 0.50 & 11.40 & 11.40 & 11.40 & 16.74 & 11.74 & 11.30 \\
\hline \hline 2.0 & 0.50 & -0.50 & 23.20 & 23.20 & 23.10 & 26.28 & 31.47 & 23.00 \\
\hline 2.0 & 0.50 & 0.50 & 18.50 & 18.50 & 18.70 & 18.85 & 20.48 & 18.30 \\
\hline 2.0 & 2.00 & -0.50 & 11.10 & 11.10 & 11.10 & 17.70 & 11.63 & 11.00 \\
\hline 2.0 & 2.00 & 0.50 & 10.90 & 10.90 & 10.90 & 15.46 & 11.03 & 10.70 \\
\hline \hline 3.0 & 0.50 & -0.50 & 46.80 & 46.80 & 45.60 & 46.12 & 51.67 & 46.30 \\
\hline 3.0 & 0.50 & 0.50 & 11.60 & 11.60 & 11.90 & 10.81 & 9.80 & 11.40 \\
\hline 3.0 & 2.00 & -0.50 & 11.60 & 11.60 & 11.60 & 17.81 & 12.44 & 11.50 \\
\hline 3.0 & 2.00 & 0.50 & 10.20 & 10.20 & 10.30 & 13.72 & 10.12 & 10.10 \\
\hline
\end{tabular}


for $\zeta=0.5-0.5 i$ as the Mach number is increased (see Tables 3-5). CH2DDS, LEE2DDS and CH3DDS remain in good agreement with the mode theory results (note that at $2.0 \mathrm{kHz}$ that LEE2DDS is in good agreement with the mode theory results now that the peak attenuation is much lower). Also, given the low fidelity of CH3DPA compared to the other codes, its predicted attenuation is good, except at a few selected frequencies where wide-angle effects are more pronounced. Finally, the attenuation of LEE2DIS is diverging from the attenuation predicted by mode theory and from the attenuation predicted by other codes at these Mach numbers. This is an unexpected result but appears due to LEE2DIS nonreflecting boundary condition that is less accurate for the nonplanar wave source used in this second example problem. Note that LEE2DIS and LEE3DIS are in good agreement with exact results for the planar source example presented in Table 1.

Our first example problem contained a hardwall and the second example contained a uniform liner. In these two example problems, the inputs to the prediction codes were known and exact or approximate solutions for the attenuations were determined analytically for comparison with code predictions. A third example is now presented for which the lower wall, two sidewalls, and portions of the upper wall are rigid. A $0.41 \mathrm{~m}$-long, uniformly treated section is positioned $0.10 \mathrm{~m}$ downstream of the source plane. The test liner was a conventional perforate-over-honeycomb material whose grazing flow impedance was obtained from measurements in the GIT. This configuration is referenced in the following discussion as the segmented liner example. To avoid reflections from the trailing edge of the segmented liner, the test section length was increased to $0.711 \mathrm{~m}$ and the grid spacing was kept at that of the previous examples by increasing the grid density to a $113 \times 21$ evenly spaced grid. Unfortunately, approximate analytical expressions for the segmented liner attenuation are not available. Predicted attenuations are therefore compared to measured data. Additionally, because the code inputs must be measured, they are not known exactly but are subject to measurement uncertainty. Uncertainty also exists in the measured attenuation. Therefore, measurement and predictions are compared on a statistical basis.

For the segmented liner, attenuation predictions are performed for a $140 \mathrm{~dB}$ plane wave source in the GIT, in which the upper wall contains a $0.40 \mathrm{~m}$-long liner. Results were acquired at three flow rates for frequencies of $1.0,2.0$ and $3.0 \mathrm{kHz}$. Data were acquired at each of these conditions at least eight times in order to allow for a small-sample statistical evaluation of code inputs and measured attenuation. These tests were conducted at different times over a period of one year so as to minimize systematic errors due to atmospheric effects. The mean values of the following parameters were computed from these measurements: (1) source SPL, (2) average Mach number, (3) liner impedance, (4) exit impedance, (5) static pressure, and (6) static temperature. Each of these mean values were used as inputs to the duct propagation codes, and the predicted attenuations were compared to the mean of the measured attenuations.

Table 6 Comparison of measured and predicted liner attenuation for three selected codes using a segmented liner

\begin{tabular}{|c|c|c|c|c|c|}
\hline Mach & $\mathrm{kHz}$ & Measured & CH2DDS & LEE2DDS & CH3DPA \\
\hline 0.00 & 1.0 & 9.52 & 10.25 & 10.43 & 12.08 \\
\hline 0.00 & 2.0 & 7.44 & 8.14 & 8.23 & 22.30 \\
\hline 0.00 & 3.0 & 2.03 & 2.03 & 2.07 & 1.57 \\
\hline 0.25 & 1.0 & 8.55 & 11.42 & 11.25 & 14.88 \\
\hline 0.25 & 2.0 & 23.54 & 23.08 & 24.08 & 22.29 \\
\hline 0.25 & 3.0 & 6.06 & 5.85 & 5.74 & 5.67 \\
\hline 0.40 & 1.0 & 5.57 & 7.53 & 3.81 & 9.59 \\
\hline 0.40 & 2.0 & 14.44 & 13.95 & 13.93 & 13.06 \\
\hline 0.40 & 3.0 & 7.95 & 7.12 & 6.99 & 7.31 \\
\hline
\end{tabular}

Table 6 compares the predicted code attenuations (using the mean value for the code input parameters) with the average attenuation from the measurements. As observed in Table 6, the mean of the measured attenuation is in generally good agreement with code predictions with one exception. Note that the code that uses the parabolic approximation (i.e., CH3DPA) does not agree well with the measurement at $2.0 \mathrm{kHz}$ and Mach zero. Among the most likely sources of the discrepancy between the CH3DPA result and measurement (i.e., at $2.0 \mathrm{kHz}$ ) are reflections from the leading or trailing edge of the liner, or the generation of wide-angle modes in the duct. Both of these effects are neglected by the low fidelity code, CH3DPA. 


\section{B. Efficiency Assessment}

Two important metrics often used to determine the efficiency of a computer code are wall clock time and computer memory requirements. The suite of codes presented in this study solve a discrete set of acoustic equations using either a direct solve (e.g., CH2DDS, LEE2DDS, CH3DPA, CH3DDS, LEE3DDS) or an iterative solve (e.g., LEE2DIS, LEE3DIS) strategy to compute the acoustic solution. The codes that are based on an iterative solve strategy were designed for computers with low memory, whereas those that implement direct solve strategies are designed for high memory machines (with the exception of CH3DPA). Generally speaking, the wall clock time is not as important on low memory machines because they are often under the control of a single user and there is little competition for wall clock time from other users. On the other hand, high memory machines often support many users that compete for available wall clock time. In this section, we choose to place focus on how these codes are used at the NASA Langley Research Center (LaRC), where the most important efficiency metric for deciding on the choice of a code is the wall clock time. This occurs for three primary reasons: (1) codes are often run on a massively-parallel computer within a multi-user environment where users must compete for wall clock time, (2) much emphasis is placed on impedance eduction, in which the selected propagation code must be run hundreds (sometimes thousands) of times to educe the unknown impedance of a test liner, and (3) LaRC is working toward the ability to model full-scale, large commercial engine nacelles with high fidelity codes to get a quality prediction (high fidelity codes require significantly more wall clock time than low fidelity codes). Again, it is reemphasized that other research organizations may utilize these codes for other reasons or may have different computing facilities that may dictate choosing a different metric for analyzing code efficiency.

Table 7. Wall clock time consumed on Columbia clusters by in-duct codes on baseline grid

\begin{tabular}{|l|c|l|l|c|c|}
\hline $\begin{array}{c}\text { Code } \\
\text { Name }\end{array}$ & $\begin{array}{c}\text { No. of } \\
\text { Dims }\end{array}$ & $\begin{array}{c}\text { Governing } \\
\text { Equations }\end{array}$ & \multicolumn{1}{|c|}{$\begin{array}{c}\text { Numerical } \\
\text { Method }\end{array}$} & $\begin{array}{c}\text { No. of } \\
\text { CPUs }\end{array}$ & $\begin{array}{c}\text { Wall } \\
\text { clock }\end{array}$ \\
\hline CH2DDS & 2 & Convected Helmholtz & Cubic Finite Element & 8 & $48 \mathrm{~s}$ \\
\hline LEE2DDS & 2 & Linearized Euler & Linear Finite Element & 8 & $60 \mathrm{~s}$ \\
\hline LEE2DIS & 2 & Linearized Euler & Pseudo Time-Stepping & 1 & $210 \mathrm{~s}$ \\
\hline CH3DPA & 3 & Convected Helmholtz & Parabolic Approximation & 1 & $7 \mathrm{~s}$ \\
\hline CH3DDS & 3 & Convected Helmholtz & Cubic Finite Element & 32 & $912 \mathrm{~s}$ \\
\hline LEE3DIS & 3 & Linearized Euler & Pseudo Time-Stepping & 4 & $5400 \mathrm{~s}$ \\
\hline
\end{tabular}

Table 7 gives the wall clock time on the baseline grid for the various codes. The six columns in the table correspond to the code name, number of spatial dimensions, governing differential equations, the type of numerical method, the number of CPUs used, and the wall clock time in seconds. Although the wall clock times are those to obtain the solution in the hardwall duct, there was little change in the wall clock time when the wall was lined with acoustic material. As should be expected, the two codes that use direct solve strategies (CH2DDS, LEE2DDS) and run on more than one CPU require considerably less wall clock time than LEE2DIS, which employs an iterative solve strategy and is sequential. It is also noted that the codes using a direct solve strategy (CH2DDS, CH3DDS, LEE2DS) can be run in parallel to further reduce the wall clock time given in Table 7, although they do not scale linearly with the number of CPUs. Table 7 shows that the wall clock time increases by a factor of 19 when the cubic finite element analysis (i.e., with a direct solve strategy) is increased from 2D (CH2DDS) to 3D (CH3DDS). Note that the number of CPUs had to be increased from eight to thirty-two to allow enough memory for the CH3DDS code to run (i.e., when compared to CH2DDS) and a nineteen-fold increase in wall clock time is still observed. In contrast, Table 7 shows that the wall clock time increases by a factor of 26 when the pseudo time-stepping code (i.e., with an iterative solve strategy) is increased from 2D (LEE2DIS) to 3D (LEE3DIS). It should be noted that increasing the number of CPUs for the LEE3DIS code beyond the four in Table 7 leads to an increase in wall clock time over that in Table 7. As expected, the lowest fidelity code in the suite, CH3DPA (CH3DPA; uses the parabolic approximation), is the most time efficient. The sequential code, CH3DPA, is observed to be more than two orders of magnitude faster than CH3DDS and nearly three orders of magnitude faster than LEE3DIS. 


\section{Summary and Concluding Remarks}

Suite of seven of the most commonly used duct propagation codes (three vortical and four non-vortical) has been evaluated in this paper. The suite consists of the most commonly used codes and methods. These codes support propagation through acoustically treated inlet and aft-fan ducts in the presence of mean flow and, collectively, represent the state-of-art in the liner industry. The following is a brief summary of what was achieved in this study:

1. The basic assumptions, governing differential equations, boundary conditions, and numerical methods underlying each code development have been quantified.

2. Each code has been evaluated on the same problems, on the same computer, and using identical grids.

3. The predicted attenuations in a hardwall duct were computed for nine combinations of frequencies and Mach numbers, and were compared to known values determined from analytical models.

4. The predicted attenuations (for each code) over a section of an infinitely long duct with a uniform impedance liner along the top wall were computed for 36 combinations of Mach number, frequency, and lining impedance, and were compared to values obtained from mode theory.

5. As part of this evaluation, attenuation and code input parameters were measured eight different times over a period of one year using the same conventional perforate-over-honeycomb liner in the NASA Langley Grazing Incidence Tube (GIT). The measured code inputs were then averaged and used as input for code attenuation predictions. These predictions were compared to the measured mean attenuations for a $140 \mathrm{~dB}$ incident sound pressure level, for three flow rates, and source excitation frequencies of 1.0, 2.0, and $3.0 \mathrm{kHz}$.

The following primary findings have resulted:

1. Wall clock time increases of an order of magnitude or more are observed for $3 \mathrm{D}$ codes relative to the corresponding 2D versions of the same codes. As should be expected, codes using an iterative solve methodology are considerably more memory efficient and less time efficient than those based upon a direct solve methodology.

2. Attenuations predicted with each code are in good agreement with exact results for the hardwall duct.

3. Attenuations predicted with the CH2DDS, LEE2DDS, CH3DDS, and CH3DPA codes over a portion of an infinitely-long duct with uniform acoustic treatment compare well with results from mode theory for the 36 combinations of Mach number, frequency, and wall impedance used in this study. Of these four codes, the CH3DPA is considerably more time efficient. The LEE2DIS attenuation predictions compare well with mode theory at low Mach numbers, but tend to diverge from both mode theory and other code results as the flow Mach number is increased. Only the finite element simulation of the convected Helmholtz equation using cubic Hermite polynomials was able to accurately predict extremely large liner attenuations.

4. Attenuations predicted for a conventional perforate-over-honeycomb liner installed in the GIT compare well with measured attenuations when compared on a statistical basis.

This investigation has provided valuable insights with regards to strengths and weakness of several duct propagation codes and is expected to form the basis for continuing code development at NASA Langley Research Center.

\section{References}

${ }^{1}$ Dougherty, R. P., "A Wave-Splitting Technique for Nacelle Acoustic Propagation,"Proceedings of the 3rd AIAA/CEAS Aeroacoustic Conference, 1997, pp.550-556.

${ }^{2}$ Free-Field-Technologies-S.A. Actran 2006 Aeroacoustic Solutions, “Actran/TM and Actran/LA - User’s Manual.” 16, place de l’ Universite, 1348 Louvain-la-Neuve, Belgium, 2006

${ }^{3}$ Lan, J. H., and Breard, c., "Development and Validation of a 3D Linearized Euler Solver," AIAA Paper 2006-2585, Proceedings of the 12th AIAA/CEAS Aeroacoustics Conference \& Exhibit, May 8-10, 2006, Cambridge, MA.

${ }^{4}$ Watson, W. R. and Jones, M. G., "Comparison of Convected Helmholtz and Euler Model for Impedance Eduction in Flow," AIAA Paper 2006-2643, Proceedings of the 12th AIAA/CEAS Aeroacoustics Conference \& Exhibit, Cambridge, MA, May, 2006.

${ }^{5}$ D. Nark, W. Watson, and M. Jones, "Further Investigation of Two Acoustic Propagation Codes for Three-Dimensional Geometries," AIAA Paper 2006-2586, Proceedings of the 12th AIAA/CEAS Aeroacoustics Conference \& Exhibit, Cambridge, Massachusetts, USA, May 8 - 10, 2006.

${ }^{6}$ Watson, W., Jones, M., and Parrott, T., “ A Quasi-3-D Theory for Impedance Eduction in Uniform Grazing Flow," AIAA Paper 2005-2848, Proceedings of the 11th AIAA/CEAS Aeroacoustics Conference \& Exhibit, May 23-25, 2005, Monterey, CA. 
${ }^{7}$ Eversman, Walter, "Turbofan Acoustic Propagation and Radiation," NASA NAG3-2109, June 2000.

${ }^{8}$ Zorumski, W.E., Watson, W.R., and Hodge, S.L., "A Nonlocal Computational Boundary Condition for Duct Acoustics," Journal of Computational Acoustics, Vol. 3(1), June 1995, pp. 15-26.

${ }^{9}$ Myers, M.K., "On The Acoustic Boundary Condition In The Presence Of Flow," Journal Of Sound And Vibrations, Vol. 71(3), 1980, pp. 429-434.

${ }^{10}$ Morfey, C.L., "Acoustic Energy in Non-uniform Flows," Journal of Sound and Vibration, Vol. 14, 1971, pp. 159-170.

${ }^{11}$ Watson, W.R., Jones, M.G., and Parrott, T.L.: "Validation of an Impedance Eduction Method in Flow," AIAA Journal, Vol. 37, No. 7, July 1999, pp. 818-824.

${ }^{12}$ Nark, D., Farassat, F., Pope, D., and Vatsa, V., "The Development of the Ducted Fan Noise Propagation and Radiation Code CDUCT-LaRC," AIAA 2003-3242, 2003.

${ }^{13}$ Dougherty, R., “A Parabolic Approximation for Flow Effects on Sound Propagation in Nonuniform, Softwall, Ducts," AIAA 99-1822, 1999.

${ }^{14}$ Corones, J., "Bremmer Series that Correct Parabolic Approximations," Journal of Mathematical Analysis and Applications, Vol. 50, 1975, pp. 361-372.

${ }^{15}$ Wolfram, Stephen: "The Mathematica Book," 4th ed., Wolfram Media/Cambridge University, Champaign, ILL, 1999, chapter 1.

${ }^{16}$ Chandrakant, S. and Abel, J. F.: “Introduction to the Finite Element Method,"Van Nostrand Reinhold Company, New York, N. Y., 1972, chapter 5 .

${ }^{17}$ Thompson, K.W., "Time Dependent Boundary Conditions for Hyperbolic Systems," Journal of Computational Physics Vol. 68, pp. 1-28, 1987.

${ }^{18}$ Tam, C.K.W., and Webb, J.C., "Dispersion-Relation-Preserving Finite Difference Schemes for Computational Acoustic," Journal of Computational Physics, Vol. 107, 1993, pp. 262-282. 